\title{
PROTOCOLO DE REGENERACIÓN DE Calibrachoa var. PAMPA SALMÓN INTA A PARTIR DE MERISTEMAS APICALES
}

\author{
A REGENERATION PROTOCOL OF Calibrachoa var. PAMPA SALMÓN INTA \\ FROM APICAL MERISTEMS \\ Leticia Tombion $^{1 *}$, Adriana Kato ${ }^{1}$ y María Silvina Soto ${ }^{1}$ \\ ${ }^{1}$ Instituto Nacional de Tecnología Agropecuaria (INTA), Instituto de Floricultura, 1686, Hurlingham, \\ Buenos Aires, Argentina. \\ *Autor para correspondencia E-mail: tombion.leticia@inta.gob.ar
}

\section{RESUMEN}

Calibrachoa, perteneciente a la familia Solanaceae, es un género nativo de América del Sur. Las variedades comerciales de esta planta tienen un alto impacto en el mercado de plantas ornamentales por la atractiva forma y color de sus flores, y por su capacidad para adaptarse al uso en canteros y macetas. El objetivo de esta investigación fue desarrollar un protocolo eficiente para la propagación in vitro de Calibrachoa var. Pampa Salmón INTA a partir de meristemas apicales. La combinación de MS $1 / 2+0,01 \mathrm{mg} \mathrm{L}^{-1}$ BAP resultó en la obtención de un alto porcentaje de regeneración de plantas completas, además de una mayor producción aérea y radical que, consecuentemente, permitieron una alta tasa de sobrevivencia de plantas luego de su transferencia al invernáculo.

Palabras clave: Calibrachoa, planta ornamental, cultivo de meristemas, micropropagación, BAP, bencilaminopurina.

\section{ABSTRACT}

Calibrachoa, which belongs of the Solanaceae family, is a genus native to South America. Some commercial varieties of this plant have a high impact on the ornamental plant market because of the color and attractive shape of their flowers as well as their adaptability to be grown in flower beds and flower pots. The objective of this work was to develop an efficient protocol for in vitro propagation of Calibrachoa var. Pampa Salmon INTA from apical meristems. The combination of MS $1 / 2$ and 0.01 $\mathrm{mg} \mathrm{L}^{-1}$ BAP resulted in a high percentage of regeneration of plants in addition to a greater aerial (shoot) and radical (root) production that, consequently, allowed for a high rate of plant survival after transfer to the greenhouse.

Key words: Calibrachoa, ornamental plant, meristem culture, micropropagation, BAP, bencilaminopurine. 


\section{INTRODUCCIÓN}

Calibrachoa es un género de plantas perteneciente a la familia Solanaceae originario de América del Sur, específicamente de Brasil, Argentina y Uruguay (Tsukamoto et al., 2002), que tiene importancia económica en el mercado mundial de plantas ornamentales por su frecuente uso en canteros y macetas (Colombo et al., 2017) debido a la variedad de colores y a la atractiva forma de sus flores (Milicia et al., 2016).

La principal fuente de abastecimiento de Calibrachoa al mercado es la propagación agámica (Liu et al., 2008). Sin embargo, este método puede contribuir con la transmisión de enfermedades sistémicas que afectan la calidad y el rendimiento del cultivo (Whitfield et al., 2015). Por ello, la obtención de un método de regeneración de plantas eficiente, reproducible y que garantice la sanidad vegetal es un requisito fundamental y de carácter obligatorio por parte del Instituto Nacional de Semillas de la Argentina para el registro y la producción de variedades de calidad.

La producción de plantas in vitro permite conseguir, en condiciones asépticas de cultivo, réplicas idénticas de plantas madre en cualquier época del año y en solo la mitad de tiempo que normalmente se tomaría en el cultivo tradicional. Además, las pequeñas plantas producidas requieren un menor espacio para desarrollarse (Figueroa, 2015) y, a su vez, pueden ser saneadas de enfermedades sistémicas (Conci, 2004).

En la Argentina, el Instituto de Floricultura del Instituto Nacional de Tecnología Agropecuaria (INTA) se dedica actualmente a la domesticación y al mejoramiento genético de Calibrachoa con el propósito de obtener a partir de especies nativas, variedades ornamentales adaptadas a las condiciones del mercado local e internacional, dentro de un marco legal y sustentable del uso de los recursos (Greppi et al., 2011). Por lo tanto, el presente estudio tuvo como objetivo desarrollar un protocolo eficiente para la propagación de Calibrachoa var. Pampa Salmón INTA a partir de meristemas apicales, lo que permitirá conocer el comportamiento in vitro de esta variedad, y posibilitará en una futura investigación la limpieza de enfermedades sistémicas.

\section{MATERIALES Y MÉTODOS}

\section{Material vegetal}

Se utilizaron plantas de entre 8 y $13 \mathrm{~cm}$ de altura de Calibrachoa var. Pampa Salmón INTA conservadas en condiciones in vitro en medio de cultivo MS (Murashige y Skoog, 1962) a $25^{\circ} \mathrm{C}$ de temperatura y bajo un fotoperíodo de 16 horas de luz diarias.

\section{Preparación de explantes}

Meristemas apicales de 0,2-0,5 mm de longitud fueron extraídos mediante bisturíes y agujas de disección con la ayuda de una lupa binocular (Nikon ${ }^{\circledR}$ modelo SMZ-10, Tokio, Japón) en una cámara de flujo laminar. Se emplearon tubos de ensayo de $24,0 \times 115 \mathrm{~mm}$ que fueron completados con $10 \mathrm{~mL}$ de medio de cultivo y se introdujo un meristema por cada tubo.

\section{Tratamientos analizados}

Los meristemas fueron introducidos en diferentes medios de cultivo, conformados por un medio base más un regulador de crecimiento, de la siguiente manera: MS con el $50 \%$ de la concentración de los macroelementos (MS $1 / 2$ )-testigo; MS 1/2 + 0,01 $\mathrm{mg} \mathrm{L}^{-1}$ kinetina (KIN); MS $1 / 2+0,01 \mathrm{mg} \mathrm{L}^{-1}$ ácido naftalenacético (ANA); MS $1 / 2+0,01 \mathrm{mg} \mathrm{L}^{-1}$ 6-bencilaminopurina (BAP); MS $1 / 2+0,01 \mathrm{mg} \mathrm{L}^{-1}$ ácido indolbutírico (IBA); MS 1/2 + $0,01 \mathrm{mg} \mathrm{L}^{-1} \mathrm{ANA}+0,20 \mathrm{mg} \mathrm{L}^{-1} \mathrm{BAP}$.

Cada uno de los medios de cultivo fue ajustado a $\mathrm{pH} 5,6$ y pasado por autoclave a $121^{\circ} \mathrm{C}$ durante 17 minutos.

\section{Establecimiento de explantes}

Los tubos de ensayo que contenían los meristemas se llevaron a una cámara de cultivo durante 40 días bajo un fotoperiodo de $16 \mathrm{~h}$ de luz a una intensidad de 1000 lux y una temperatura constante de $25^{\circ} \mathrm{C}$ para promover la diferenciación de tejidos.

\section{Variables analizadas}

Finalizado el período de crecimiento en la cámara de cultivo se registraron las siguientes variables: Regeneración de planta completa (\%): № de explantes que lograron dar origen a una planta con más de una hoja verdadera, raíces y ausencia de callo/total de explantes; № hojas/ explante: cantidad de hojas obtenidas por cada explante; Longitud aérea: largo de la porción aérea del explanto, medido en $\mathrm{cm}$; Longitud radical: largo de la mayor raíz del explanto, medido en cm; Formación de callo (\%): № de callos (masa de células no diferenciadas) producidos/total de explantes; Hiperhidricidad (\%): № de explantes con aspecto vítreo o acuoso/total de explantes; Mortandad (\%): № de explantes muertos (de aspecto necrótico)/total de explantes.

\section{Aclimatación ex vitro}

A los 40 días de la siembra, los explantes que desarrollaron plántula completa fueron trasplantados a macetas de $9 \mathrm{~cm}$ de diámetro que contenían sustrato compuesto a base de turba de musgoSphagnum, compost de corteza fina, perlita y cenizas (tabaco Growmix ${ }^{\circledR}$ ), y cubiertos con 
bolsas de polietileno transparentes para afrontar el período de adaptación en un invernáculo de aclimatación. Transcurridos 10 días se retiraron gradualmente las bolsas y las plantas fueron llevadas al invernadero de producción. A partir de ello se calculó la sobrevivencia ex vitro (\%): № de plantas que sobrevivieron luego de la aclimatación/total de plantas aclimatadas.

\section{Análisis estadístico}

El experimento se realizó mediante un diseño completamente aleatorizado. Se emplearon 16 explantos por tratamiento y el experimento se realizó por triplicado. Por no cumplirse los supuestos de normalidad, las variables regeneración de planta completa, formación de callo, hiperhidricidad y mortandad fueron analizadas mediante la prueba de contraste de varianzas chi-cuadrado a partir del software estadístico INFOSTAT (Di Rienzo et al., 2009). Se corroboró la normalidad de los datos para las variables producción aérea y radical, por lo que se llevó a cabo un análisis de varianza con un nivel de confianza del $95 \%$ mediante el mismo software. $\mathrm{Al}$ observar diferencias significativas entre los tratamientos, se realizó la prueba de diferencia de medias de Di Rienzo, Guzman y Casanoves-DGC$(p \leq 0,05)$ (Di Rienzo et al., 2009).

\section{RESULTADOS Y DISCUSIÓN}

Efecto de los tratamientos sobre la producción de plántulas completas, la formación de callo, la hiperhidricidad y la mortandad de los explantes

De acuerdo con el análisis estadístico de contraste de medias presentado en la Tabla 1, se pudo observar que la respuesta de los explantes de Calibrachoa var. Pampa Salmón INTA a los diferentes tratamientos dependió del tipo de regulador de crecimiento presente en el medio de cultivo base.

En cuanto a la regeneración de plantas completas a partir de meristemas apicales, los tratamientos testigo, MS $1 / 2+0,01 \mathrm{mg} \mathrm{L}^{-1} \mathrm{KIN}$; MS $1 / 2+0,01 \mathrm{mg} \mathrm{L}^{-1}$ ANA; MS 1/2 + 0,01 $\mathrm{mg} \mathrm{L}^{-1}$ IBA; y MS 1/2 + 0,01 mg L-1 ANA + 0,20 mg L-1 BAP, fueron estadísticamente similares entre sí, ya que obtuvieron los menores valores para este parámetro $(0,0,13,13$ y $0 \%$, respectivamente), pero fueron significativamente diferentes al tratamiento conformado por MS $1 / 2+0,01 \mathrm{mg}$ $\mathrm{L}^{-1} \mathrm{BAP}$ que pudo regenerar un $63 \%$ de plantas de Calibrachoa var. Pampa Salmón INTA. Estos valores no coinciden con los obtenidos por Kato et al. (2005), quienes lograron la mayor producción de plantas de Calibrachoa pigmaea, C. calycina y C. linearis a partir de la adición de KIN al medio MS. Tampoco coinciden con los resultados por Dill (2014), quien encontró en Calibrachoa sellowiana los mayores valores para este parámetro mediante la combinación de 0,01 mg L ${ }^{-1} \mathrm{ANA}+0,20 \mathrm{mg} \mathrm{L}^{-1} \mathrm{BAP}$. La citoquinina BAP cumple un rol fundamental en la división celular y en la diferenciación de los explantes. Además, participa en la activación del transporte de nutrientes (Figueroa, 2015), lo que podría explicar el mayor crecimiento de los explantes y el alto número de plantas completas obtenidas por el tratamiento conformado por MS $1 / 2+0,01 \mathrm{mg} \mathrm{L}^{-1}$ BAP. Por su parte, los tratamientos conformados

Tabla 1. Efecto de los tratamientos sobre la producción de plántulas completas, la formación de callo, la hiperhidricidad y la mortandad de explantes de Calibrachoa var. Pampa Salmón INTA a los 40 días del cultivo in vitro de meristemas apicales.

Table 1. Effect of the treatments on the production of whole plants, callus formation, hyperhydricity and explant mortality of Calibrachoa var. Pampa Salmon INTA 40 days after in vitro culture of apical meristems.

\begin{tabular}{|c|c|c|c|c|}
\hline Tratamiento & $\begin{array}{l}\text { Regeneración } \\
\text { planta } \\
\text { completa }(\%)\end{array}$ & $\begin{array}{l}\text { Formación } \\
\text { de callo }(\%)\end{array}$ & $\begin{array}{c}\text { Hiperhidricidad } \\
(\%)\end{array}$ & $\begin{array}{c}\text { Mortandad } \\
\text { (\%) }\end{array}$ \\
\hline MS $1 / 2$ & $0(0 / 16)$ a & $19(3 / 16)$ a & $0(0 / 16) a$ & $25(4 / 16)$ a \\
\hline $\mathrm{MS}^{1 / 2}+0,01 \mathrm{mg} \mathrm{L}^{-1} \mathrm{KIN}$ & $0(0 / 16)$ a & $69(11 / 16) b$ & $69(11 / 16) b$ & $44(7 / 16) b$ \\
\hline $\mathrm{MS}_{1 / 2}+0,01 \mathrm{mg} \mathrm{L}^{-1} \mathrm{ANA}$ & $13(2 / 16)$ a & $38(6 / 16) a b$ & $88(14 / 16) b$ & $31(5 / 16) b$ \\
\hline $\mathrm{MS}_{1 / 2}+0,01 \mathrm{mg} \mathrm{L}^{-1} \mathrm{BAP}$ & $63(10 / 16) b$ & $25(4 / 16)$ a & $0(0 / 16)$ a & $6(1 / 16)$ a \\
\hline MS $1 / 2+0,01 \mathrm{mg} \mathrm{L}^{-1} \mathrm{IBA}$ & $13(2 / 16)$ a & $0(0 / 16)$ a & $25(4 / 16)$ a & $0(0 / 16)$ a \\
\hline $\begin{array}{l}\text { MS } 1 / 2+0,01 \mathrm{mg} \mathrm{L}^{-1} \mathrm{ANA}+ \\
0,20 \mathrm{mg} \mathrm{L}^{-1} \mathrm{BAP}\end{array}$ & $0(0 / 16)$ a & $100(16 / 16) b$ & $25(4 / 16)$ a & $0(0 / 16) \mathrm{a}$ \\
\hline
\end{tabular}

Letras distintas en columnas indican diferencias significativas $(\mathrm{p} \leq 0,05)$

MS: Murashige y Skoog; KIN: kinetina; ANA: ácido naftalenacético; BAP: bencilaminopurina; IBA: ácido indolbutírico. 
por auxinas no lograron alcanzar altos valores de regeneración de plantas completas ya que, posiblemente, la presencia de este regulador de crecimiento ejerció cierta inhibición en los niveles de citoquinina endógena de cada explante por medio de los mecanismos de biosíntesis (Cervantes Flores, 2014), lo cual pudo haber dificultado los procesos de diferenciación celular y la actividad metabólica en los que intervienen las citoquininas para favorecer el crecimiento de los explantes (Figueroa, 2015).

Por otro lado, los tratamientos testigo, MS $1 / 2+0,01 \mathrm{mg} \mathrm{L}^{-1}$ BAP; y MS $1 / 2+0,01 \mathrm{mg} \mathrm{L}^{-1}$ IBA no mostraron diferencias significativas en cuanto a la formación de callo $(19 \%, 25 \%$ y $0 \%$, respectivamente), pero sí con respecto a los tratamientos MS 1/2 + 0,01 $\mathrm{mg} \mathrm{L}^{-1} \mathrm{KIN} ; \mathrm{MS}^{1 / 2}+0,01$ $\mathrm{mg} \mathrm{L}^{-1}$ ANA; y MS 1/2 + 0,01 $\mathrm{mg} \mathrm{L}^{-1} \mathrm{ANA}+0,20 \mathrm{mg}$ $\mathrm{L}^{-1} \mathrm{BAP}$ que fueron ampliamente superiores por haber formado callo en el 69, 38 y 100\% de los explantes. Tanto las auxinas como las citoquininas son capaces de inducir la producción de tejidos no organizados (Jordán y Casaretto, 2006), por lo cual se pudo ver que la mayor producción de callo se dio en estos últimos tratamientos. Estos resultados se relacionan con los obtenidos por Kato et al. (2005), quienes observaron esta continua y activa división celular desorganizada en meristemas de $C$. parviflora cultivados en un medio compuesto por $0,05 \mathrm{mg} \mathrm{L}^{-1} \mathrm{KIN}$ y $0,01 \mathrm{mg}$ $\mathrm{L}^{-1}$ ANA.

En cuanto a la hiperhidricidad, los tratamientos testigo y MS 1/2 + 0,01 $\mathrm{mg} \mathrm{L}^{-1}$ BAP no presentaron aspecto vítreo en ninguno de los casos $(0 \%)$, lo cual fue estadísticamente similar a los tratamientos MS $1 / 2+0,01 \mathrm{mg} \mathrm{L}^{-1} \mathrm{IBA}_{\text {y MS }} 1 / 2+0,01$ $\mathrm{mg} \mathrm{L}^{-1} \mathrm{ANA}+0,20 \mathrm{mg} \mathrm{L}^{-1} \mathrm{BAP}$, que presentaron vitrificación en un $25 \%$. Estos valores fueron significativamente diferentes a los alcanzados por MS 1/2 + 0,01 mg L-1 KIN; y MS 1/2+0,01 mg $\mathrm{L}^{-1} \mathrm{ANA}$, donde la hiperhidricidad se manifestó en un 69 y $88 \%$, respectivamente. Asimismo, la mayor cantidad de explantes necrosados se dio en los tratamientos MS $1 / 2+0,010 \mathrm{mg} \mathrm{L}^{-1}$ KIN y MS $1 / 2+0,010 \mathrm{mg} \mathrm{L}^{-1}$ ANA por encontrarse en un 44 y un $31 \%$ de los casos, respectivamente. El exceso de agua en el apoplasto de los explantes pudo haber dificultado el intercambio gaseoso de las células, lo cual pudo haber desencadenado su muerte (Hongyang et al., 2017), ya que los mayores valores de hiperhidricidad coincidieron con los más altos de mortandad. Por su parte, los medios testigo; MS 1/2 +0,01 $\mathrm{mg} \mathrm{L}^{-1}$ BAP; y MS $1 / 2$ $+0,01 \mathrm{mg} \mathrm{L}^{-1}$ IBA lograron los menores valores de mortandad (25, 6 y $0 \%$, respectivamente) y tuvieron diferencias estadísticas significativas a los alcanzados por los tratamientos MS $1 / 2+0,010$ $\mathrm{mg} \mathrm{L}^{-1} \mathrm{KIN}$; y MS 1/2 + 0,010 $\mathrm{mg} \mathrm{L}^{-1}$ ANA.
Efecto de los tratamientos sobre la producción aérea y radical de los explantos

Dado que los tratamientos MS $1 / 2+0,01 \mathrm{mg} \mathrm{L}^{-1}$ KIN; MS 1/2 + 0,01 $\mathrm{mg} \mathrm{L}^{-1}$ ANA; y MS 1/2 + 0,010 mg $\mathrm{L}^{-1} \mathrm{ANA}+0,200 \mathrm{mg} \mathrm{L}^{-1} \mathrm{BAP}$ desencadenaron altos valores de callo, hiperhidricidad, mortandad $y$, por consiguiente, no alcanzaron un desarrollo efectivo de plantas completas a partir de meristemas, se decidió continuar el análisis estadístico con los tratamientos MS $1 / 2+0,01 \mathrm{mg}$ $\mathrm{L}^{-1}$ BAP y MS 1/2 + 0,01 mg L ${ }^{-1}$ IBA en comparación con el tratamiento testigo.

De acuerdo con el análisis de la varianza, la producción de hojas/explante del tratamiento MS $1 / 2+0,01 \mathrm{mg} \mathrm{L}^{-1}$ BAP fue significativamente superior $(9,38)$ a la producción de los tratamientos testigo $(1,63)$ y MS 1/2+0,01 $\mathrm{mg} \mathrm{L}^{-1} \mathrm{IBA}(1,88)$, cuyos valores fueron estadísticamente similares entre sí (Fig. 1). Esta respuesta puede explicarse ya que las citoquininas intervienen en la organogénesis vegetal, por lo tanto, inducen la formación de hojas y brotes (Figueroa, 2015), tal como sucedió en el tratamiento conformado por MS $1 / 2+0,01 \mathrm{mg}$ $\mathrm{L}^{-1}$ BAP que obtuvo el mayor número de hojas/ explante. Estos resultados no concuerdan con los observados por Dill (2014), quien encontró que la mayor producción de brotes de C. sellowiana se dio en un medio de cultivo MS suplementado con $0,01 \mathrm{mg} \mathrm{L}^{-1} \mathrm{ANA}+0,20 \mathrm{mg} \mathrm{L}^{-1} \mathrm{BAP}$.

En cuanto a la altura de los explantes se observó la misma tendencia que para la variable № de hojas/explante, donde se vio que el tratamiento MS $1 / 2+0,01 \mathrm{mg} \mathrm{L}^{-1}$ BAP obtuvo la mayor longitud aérea $(0,71)$, significativamente diferente al tratamiento testigo $(0,11)$ y al tratamiento $\mathrm{MS}^{1} / 2+0,01 \mathrm{mg} \mathrm{L}^{-1} \mathrm{IBA}(0,19)$ (Fig. 2). Tal como ya se mencionó, las citoquininas juegan un rol importante en varias fases del desarrollo de las plantas. Ello involucra la desinhibición y diferenciación de yemas, el alargamiento celular y el flujo de asimilados y nutrientes, por lo que producen mayor cantidad de ramificaciones y mayor longitud aérea de las plantas (Lagoutte et al., 2009). Por ello, se asume que el tratamiento compuesto por MS $1 / 2+0,01 \mathrm{mg} \mathrm{L}^{-1}$ BAP pudo diferenciarse de los tratamientos restantes. Estos valores tampoco coinciden con los observados por Dill (2014), que observó la mayor altura de explantes de $C$. sellowiana en un tratamiento conformado por MS $+0,01 \mathrm{mg} \mathrm{L}^{-1}$ ANA y otro compuesto por MS sin la adición de reguladores de crecimiento.

En el análisis de la longitud radical se vio que no hubo diferencias estadísticas significativas entre ninguno de los tratamientos observados $(0$; 0,38 y 0,59 ) (Fig. 3). Se sabe que tanto las auxinas como las citoquininas intervienen en la formación de raíces (Bustamante et al., 2012). Sin embargo, 


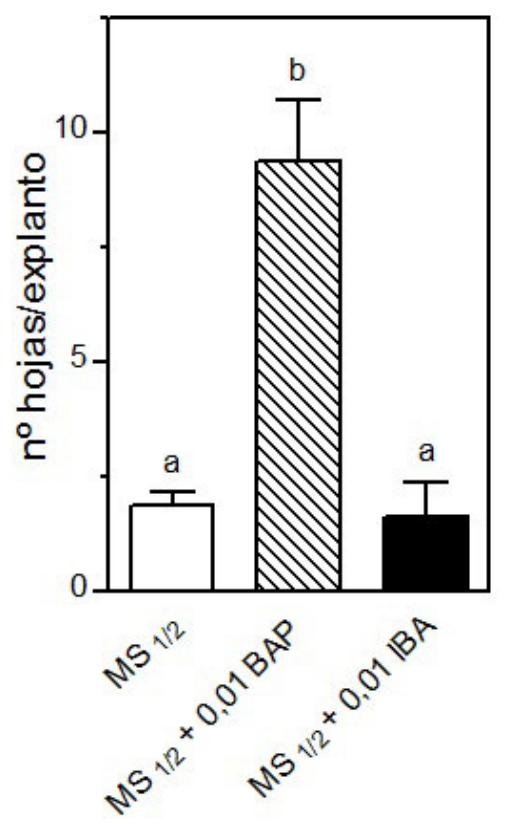

Fig. 1. Efecto de los tratamientos sobre el número de hojas/explanto.

Fig. 1. Effect of the treatments on the number of leaves / explant.

MS: Murashige y Skoog; BAP: bencilaminopurina; IBA: ácido indolbutírico.

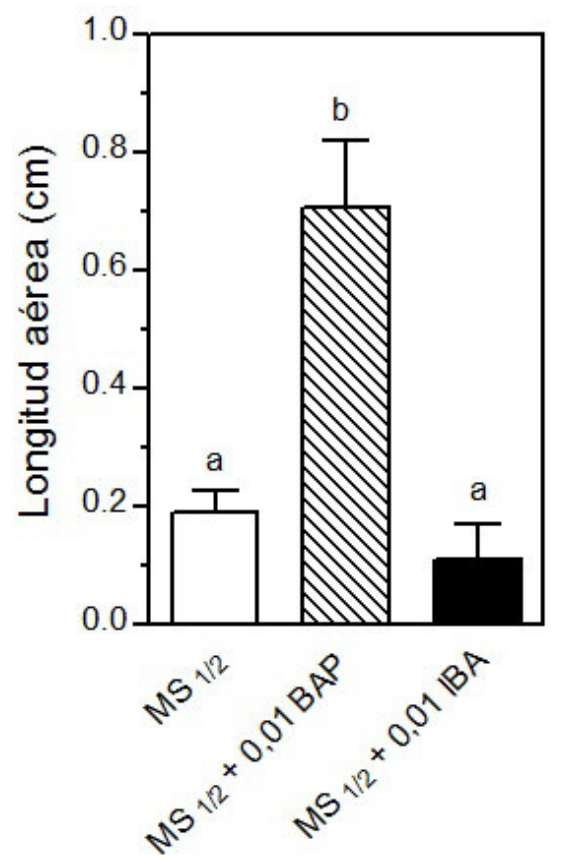

Fig. 2. Efecto de los tratamientos sobre la longitud aérea de los explantos. Fig. 2. Effect of the treatments on shoot length of the explants. 


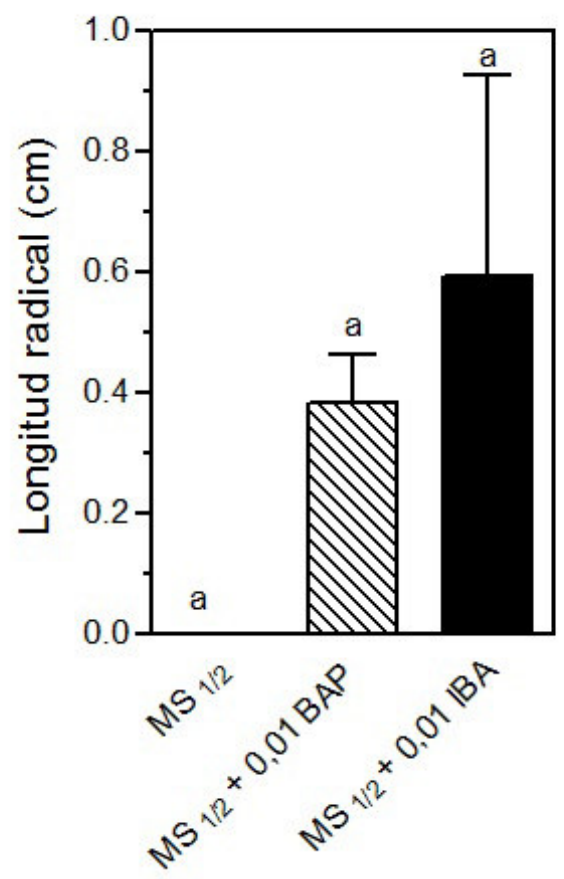

Fig. 3. Efecto de los tratamientos sobre la longitud radical de los explantos. Fig. 3. Effect of treatments on root length of the explants.

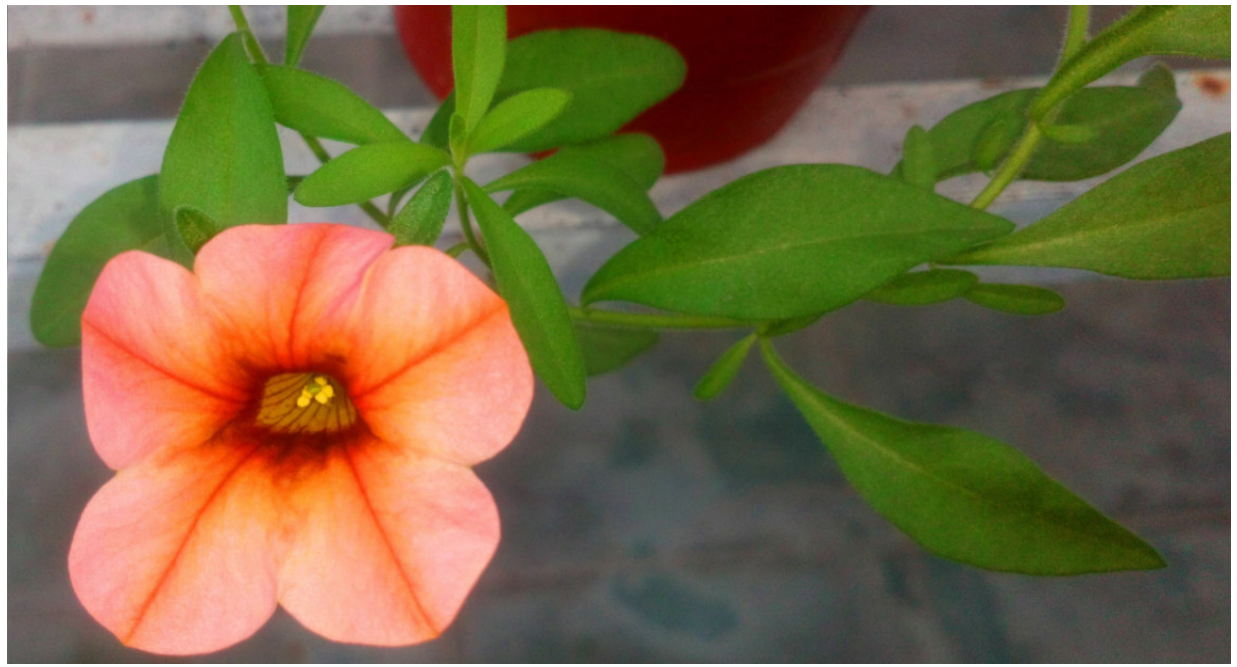

Fig. 4. Calibrachoa var. Pampa Salmón INTA regenerada a partir de meristemas apicales mediante MS $1 / 2+0,01 \mathrm{mg} \mathrm{L}^{-1}$ BAP.

MS: Murashige y Skoog; BAP: bencilaminopurina.

Fig. 4. Calibrachoa var. Pampa Salmon INTA regenerated from apical meristems using MS $1 / 2+0.01$ $\mathrm{mg} \mathrm{L}^{-1}$ BAP. 
la baja producción radical de los tratamientos conformados por MS $1 / 2+0,01 \mathrm{mg} \mathrm{L}^{-1}$ IBA y MS 1/2 $+0,01 \mathrm{mg} \mathrm{L}^{-1} \mathrm{BAP}$ pudo deberse a que el tiempo que el explante estuvo en contacto con el medio de cultivo no fue el adecuado para lograr un alto desarrollo de raíces. Por su parte, Kato et al. (2005) encontraron que los mayores valores de crecimiento de raíces de $C$. parviflora se dieron en un medio compuesto por MS + 0,01 mg $\mathrm{L}^{-1} \mathrm{KIN}$, lo cual difiere notablemente de los resultados alcanzados durante este experimento. Tal como se vio, tanto para la producción aérea como para la radical, el tratamiento testigo (MS1/2) no logró alcanzar valores significativamente superiores, ya que la ausencia de reguladores de crecimiento en su composición no permitió que los meristemas pudieran lograr su correcta diferenciación.

\section{Sobrevivencia ex vitro de las plántulas}

Finalizado el período de adaptación se vio que el 100\% de las plantas transferidas al invernáculo (aquellas provenientes del tratamiento compuesto por MS $1 / 2+0,01 \mathrm{mg} \mathrm{L}^{-1}$ BAP) sobrevivieron al trasplante (Fig. 4), valor óptimo para la producción de plantas a partir de meristemas.

\section{CONCLUSIONES}

Se pudo determinar que la combinación del medio de cultivo base MS $1 / 2$ con $0,01 \mathrm{mg} \mathrm{L}^{-1}$ BAP resultan en un método eficiente para la propagación de Calibrachoa var. Pampa Salmón INTA a partir de meristemas apicales, ya que su empleo posibilita la obtención de un mayor número de plantas completas regeneradas $y$, además, de una mayor producción aérea y radical que, en consecuencia, permiten una alta tasa de sobrevivencia de plantas luego de su transferencia al invernáculo.

\section{AGRADECIMIENTOS}

Se agradece especialmente al Ing. Agr. Nicolás Pablo Borrelli del Instituto de Floricultura del INTA por brindar sus conocimientos estadísticos para hacer posible el análisis realizado durante esta investigación.

\section{LITERATURA CITADA}

Bustamante, G., J. Imata, L. Linares, D. Mostajo, R. Pacheco, y A. Vilca. 2012. Efectos de las fitohormonas (auxinas, giberelinas $\mathrm{y}$ citoquininas) en el crecimiento de hipocotilos de Caesalpinea spinosa (Molina) Kuntze ‘Tara'. Curso de Fisiología Vegetal. Universidad Nacional de San Agustín, Arequipa, Perú.
Cervantes Flores, F. 2014. Evaluación de efectos de resistencia-susceptibilidad a plagas y enfermedades, productividad y calidad frente a la aplicación de citoquininas y auxinas en plantas de rosa (Rosa sp.). Trabajo de graduación para acceder al título de Ingeniero en Agroempresa. Universidad San Francisco de Quito, Ecuador.

Colombo, N., A. Coviella, and J. C. Hagiwara. 2017. A novel source of cytoplasmatic male sterility in Calibrachoa pubescens. Ornamental Horticulture 23(3):311-318.

Conci, V. 2004. Obtención de plantas libres de virus. Biotecnología y Mejoramiento Vegetal. Capítulo 5. Ediciones INTA, Buenos Aires, Argentina.

Di Rienzo, J.A., F. Casanoves, M.G. Balzarini, L. Gonzalez, y M. Tablada. 2009. Grupo Infostat. Facultad de Ciencias Agrarias, Universidad Nacional de Córdoba, Argentina.

Dill, L. 2014. Estrategias de micropropagación de Calibrachoa sellowiana (SENDTN.) Wijsman y Rosmarinus officinalis L. Trabajo de graduación en Agronomía para acceder al título de Ingeniero Agrónomo. Universidad Federal de Santa Catarina, Santa Catarina, Brasil.

Figueroa, R.F. 2015. Efectos de diferentes dosis de citoquinina en interacción con un compuesto orgánico en la germinación in vitro de semilla de moringa (Moringa oleifera Lam.) Trabajo de graduación en Agronomía para acceder al título de Ingeniero Agrónomo. Universidad Técnica de Machala, Unidad Académica de Ciencias Agrarias, Ecuador.

Greppi, A, J.C. Hagiwara, y J. Stehmann. 2013. Novedades en Calibrachoa (Solanaceae) y notas taxonómicas sobre el género para la Argentina. Darwiniana 1(1):173-187.

Hongyang, G., X. Xiuying, A. Lijian, X. Xin, and L. Yuan. 2017. Reversion of hyperhydrycity in pink (Dianthus chinensis L.) plantlets by $\mathrm{AgNO}_{3}$ and its associated mechanism during in vitro culture. Plant Science 254:1-11.

Jordán, M., y J. Casaretto. 2006. Fisiología vegetal. Capítulo XV. Hormonas y reguladores de crecimiento: auxinas, giberelinas y citocininas. Ediciones Universidad de La Serena, La Serena, Chile.

Kato, A.E., J.C Hagiwara, M.N. Mori, and M.S. Soto. 2005. Meristem culture for the conservation and propagation in four Calibrachoa species native to Argentina. XV Congresso Brasileiro de Floricultura e Plantas Ornamentales y II Congreso Brasileiro de Cultivo de Tejidos de Plantas. 7-12 de agosto 2005. Libro de resúmenes. 644 p. Asociación Brasilera de Horticultura, Fortaleza, Brasil. 
Lagoutte, S.M., M. Divo, y F. Vilella. 2009. Efecto del tamaño de celdas y citoquininas en el crecimiento de plantas de petunia. Revista Internacional de Botánica Experimental 78:31-36.

Liu, H., J. Sears, and R. Morrison. 2008. Isolation and characterization of a Carmo-like virus from Calibrachoa plants. Plant Disease 87(2):167-171.

Milicia, V.J., A. Coviella, M.S. Soto, y A. Chiesa, 2016. Mejoramiento de plantas nativas sudamericanas: altas temperaturas como herramienta para superar barreras precigóticas en el complejo CalibrachoaNierembergia. Chilean Journal of Agricultural \& Animal Sciences 32(2):85-93.
Murashige, T., y F. Skoog. 1962. A revised medium for rapid growth and bio assays with tobacco tissue cultures. Physiologia Plantarum 15:473-497.

Tsukamoto, T., T. Ando, H. Watanabe, H. Kokubun, G. Hashimoto, U. Sakazaki, et al. 2002. Differentiation in the status of self-incompatibility among Calibrachoa species (Solanaceae). Journal Plant Research 115:185-193.

Whitfield, A., B. Falk, and D. Rotenberg. 2015. Insect vector-mediated transmission of plant viruses. Virology 2015:278-289. doi: 10.1016/j. virol.2015.03.026 